\title{
Interactions with p300 enhance transcriptional activation by the PDZ-domain coactivator Bridge-1
}

\author{
Jee H Lee, Jamie L Volinic, Constanze Banz, Kwok-Ming Yao ${ }^{1}$ \\ and Melissa K Thomas
}

Laboratory of Molecular Endocrinology and Diabetes Unit, Massachusetts General Hospital and Harvard Medical School, Boston, Massachusetts, USA
'Department of Biochemistry, Faculty of Medicine, University of Hong Kong, Pokfulam, Hong Kong SAR, China
(Requests for offprints should be addressed to M K Thomas, Laboratory of Molecular Endocrinology and Diabetes Unit, Massachusetts General Hospital,
Wellman 340,50 Blossom Street, Boston, MA 02114, USA; Email: mthomas1@partners.org)

\begin{abstract}
Transcriptional coactivators are essential mediators of signal amplification in the regulation of gene expression in response to hormones and extracellular signals. We previously identified Bridge-1 (PSMD9) as a PDZ-domain coregulator that augments insulin gene transcription via interactions with the basic helix-loop-helix transcription factors E12 and E47, and that increases transcriptional activation by the homeodomain transcription factor PDX-1. In these studies, we find that transcriptional activation by Bridge-1 can be regulated via interactions with the histone acetyltransferase and nuclear receptor coactivator p300. In transfection assays, transcriptional activation by Bridge- 1 is increased by the inhibition of endogenous histone deacetylase activity with trichostatin $\mathrm{A}$, indicating that the transcriptional activation function of Bridge- 1 can be regulated by histone modifications. The exogenous expression of p300 enhances the transcriptional
\end{abstract}

activation by Bridge- 1 in a dose-dependent manner. In contrast, the sequestration of $\mathrm{p} 300$ by the overexpression of the adenoviral protein E1A, but not by an E1A mutant protein that is unable to interact with $\mathrm{p} 300$, suppresses the transcriptional activation by Bridge- 1 . We demonstrate that p300 and Bridge-1 proteins interact in immunoprecipitation and glutathione-S-transferase (GST) pull-down assays. Bridge- 1 interacts directly with multiple regions within $\mathrm{p} 300$ that encompass $\mathrm{C} / \mathrm{H} 1$ or $\mathrm{C} / \mathrm{H} 2$ cysteine- and histidine-rich protein interaction domains and the histone acetyltransferase domain. Deletion or point mutagenesis of the Bridge-1 PDZ domain substantially reduces transcriptional activation by Bridge- 1 and interrupts interactions with p300. We propose that p300 interactions with Bridge-1 can augment the transcriptional activation of regulatory target genes by Bridge- 1 .

Journal of Endocrinology (2005) 187, 283-292

\section{Introduction}

Transcriptional coregulators provide important regulatory flexibility in the cellular responsiveness to hormones and extracellular signals. The physiologic importance of coregulator function is highlighted by the association of coactivator dysfunction with multiple human disease states, including neurodegeneration, malignancies and metabolic disorders. The nuclear receptor coactivator p300 functions to assemble multimolecular transcriptional regulatory protein complexes through interactions with a large repertoire of transcription factors and components of basal transcription machinery (Chan \& La Thangue 2001, Vo \& Goodman 2001). The intrinsic acetyltransferase activity of p300 augments the activation of gene transcription through acetylation of histones and transcription factors (Vo \& Goodman 2001). Mutations in the human p300 gene, like those in the related Creb-binding protein (CBP) gene, result in heritable tumors in
Rubinstein-Taybi syndrome (Giles et al. 1998, Roelfsema et al. 2005). Somatic mutations in p300 also occur in sporadic tumors, consistent with the demonstrated function of this coactivator as a tumor suppressor in mouse models (Chan \& La Thangue 2001, Iyer et al. 2004). Altered levels of expression of p300 modify embryonic development, cellular functions and responsiveness to extracellular signals (Yao et al. 1998). For example, in Paget's disease, the hyperresponsiveness of osteoclast precursors to vitamin $\mathrm{D}$ is associated with increased expression levels of coactivators, including p300 (Kurihara et al. 2004). In a mouse model of Huntington's disease, progressive deficits in insulin production are correlated with decreased expression levels of p300 and other transcription factors in insulin-expressing cells (Andreassen et al. 2002).

In early-onset, autosomal-dominant heritable forms of diabetes known as maturity-onset diabetes of the young (MODY), abnormal interactions between mutant MODY transcription factors and p300 may contribute to deficits in 
insulin production and the pathogenesis of disease. p300 recruitment to the proximal insulin promoter in insulinproducing pancreatic beta cells is associated with hyperacetylation and transcriptional activation (Chakrabarti et al. 2003), and p300 interactions with the transcriptional regulators PDX-1 (pancreas duodenum homeobox-1), NeuroD1/Beta-2 and E47 enhance insulin gene transcription (Qiu et al. 1998, 2002, Sharma et al. 1999, Mosley et al. 2004, Stanojevic et al. 2004). Five direct or indirect transcriptional regulators of insulin gene expression are encoded by genes associated with MODY (Fajans et al. 2001). MODY1 mutations R154X and E276Q in the HNF-4 $\alpha$ gene interfere with p300 recruitment and transcriptional activation (Eeckhoute et al. 2001), the MODY4 mutation P63 fsdelC results in a truncated cytoplasmic PDX-1 protein with the capacity to sequester p300 (Stanojevic et al. 2004), and the MODY6 mutation 206+C disrupts NeuroD1/Beta-2 binding to p300 (Malecki et al. 1999).

In studies designed to identify additional transcriptional regulators of the insulin promoter, we identified a PDZdomain coactivator designated Bridge-1 (Thomas et al. 1999). Although Bridge- 1 is expressed in a range of tissues, it is expressed within the pancreas predominantly in insulin-expressing pancreatic beta cells (Thomas et al. 1999). Bridge- 1 interacts with basic helix-loop-helix transcription factors E12 and E47 via a PDZ protein interaction domain and augments E47-dependent activation of glucose-responsive enhancers within the insulin promoter. The PDZ protein interaction domain within Bridge-1 has atypical features with the highest region of homology to other mammalian PDZ-domain-containing proteins concentrated between amino acids 138-178 (Thomas et al. 1999). Transcriptional activation by PDX-1 can be increased by the addition of Bridge-1 (Stanojevic et al. 2005). Endogenous Bridge-1 expression in insulinexpressing cells regulates the transcriptional activation of the insulin gene as indicated by the substantial inhibition of insulin promoter activation by Bridge-1 antisense constructs (Thomas et al. 1999). Dose-dependent increases in Bridge-1 expression regulate insulin gene expression in a biphasic pattern, and pancreatic overexpression of Bridge- 1 in transgenic mice results in insulin deficiency and diabetes (Volinic et al. 2006). Here we demonstrate that the PDZ domain of Bridge- 1 is required for transcriptional activation, and identify p300 as a Bridge-1 interaction partner that augments transcriptional activation by Bridge-1 in a PDZ-domain-dependent manner.

\section{Materials and Methods}

\section{Cell culture and transfections}

BHK-21 (C-13) cells were obtained from American Type Culture Collection (Manassas, VA, USA) and cultured in Dulbecco's modified Eagle's medium supplemented with $10 \%$ fetal bovine serum, $100 \mathrm{U} / \mathrm{ml}$ penicillin $\mathrm{G}$ and $100 \mu \mathrm{g} / \mathrm{ml}$ streptomycin sulfate (Invitrogen Life Technologies, Carlsbad, CA, USA). Transfections were conducted with Lipofectamine or Lipofectamine 2000 (Invitrogen Life Technologies) according to published methods (Thomas et al. 1999). Yeast transcriptional activation assays were conducted as previously reported (Golemis et al. 1994, Thomas et al. 1999) with quantitative LexA-operator-responsive beta-galactosidase reporter assays, as outlined by the manufacturer (BD Biosciences Clontech, Palo Alto, CA, USA).

\section{Plasmids and mutagenesis}

The Gal4 DNA-binding domain Bridge-1 fusion protein expression vectors Gal4-Bridge-1, Gal4-Bridge-1(1-72) and Gal4-Bridge-1(1-133) were generated in the pM vector (BD Biosciences Clontech) as previously described (Thomas et al. 1999). Additional mutant Gal4 Bridge-1 fusion protein expression vectors were generated by site-directed mutagenesis of the pM-Bridge-1 plasmid. Site-directed mutagenesis was performed with the QuikChange site-directed mutagenesis kit (Stratagene, La Jolla, CA, USA), according to the manufacturer's instructions, by using the following oligonucleotides and their respective reverse complements:

- Gal4-Bridge-1(V159P): 5'-TGGATGATGAAATT CCGGAGTTCGGCTCTGT-3'

- Gal4-Bridge-1(V164P): 5'-TGGAGTTCGGCTCT CCGAACACCCAAAACTT-3'

- Gal4-Bridge-1(V175P): 5'-AGTCTCTGCAGAAC CCGGGCACTGTGGTGCA-3'

- Gal4-Bridge-1(D156P): 5'-CCTGCAAGTGGATC CTGAAATTGTGGAGTT-3'

- Gal4-Bridge-1(G151P): 5'-AGCCAGTATTGCGC CCCTGCAAGTGGATGAT-3'.

Mutant constructs were verified by automated sequencing. The glutathione-S-transferase (GST)-Bridge-1 vector was cloned by excising rat Bridge- 1 cDNA from the pM-Bridge-1 vector with BamHI and EcoRI digestion and subcloning into the multiple cloning site of the pGEX-5X-1 GST fusion protein expression vector (Amersham Biosciences, Piscataway, NJ, USA). Mutant GST-Bridge-1 expression vectors were generated by site-directed mutagenesis of the pGEX-5X-1-Bridge-1 vector by using the following oligonucleotides and their respective reverse complements:

- GST-Bridge-1(1-72): 5'-GGATTTGTATCAGGT CTGAACAGCAAGGCAC-3'

- GST-Bridge-1(1-133): 5'-CAGTCCCGCCCTAC CCTAGGCCTTTGCCAGAG-3'

- GST-Bridge-1(D156P): 5'-CCTGCAAGTGGATC CTGAAATTGTGGAGTT-3'.

Mutant constructs were verified by automated sequencing. Yeast expression vectors for LexA-Bridge-1, LexA-Bridge-1(1-72), and LexA-Bridge-1(1-132) fusion 
protein constructs have been previously described (Thomas et al. 1999). The pCMV $\beta-p 300$ plasmid was obtained from Upstate Biotechnology (Lake Placid, NY, USA). The Gal4-chloramphenicol acetyltransferase (CAT, pG5-CAT) reporter plasmid was purchased from BD Biosciences Clontech, and the Gal4-luciferase reporter plasmid (pFR-luc) was obtained from Stratagene. The pCMV-E1A wild-type and mutant E1A $(\Delta 2-36)$ plasmids were gifts from $\mathrm{R}$ Stein (Vanderbilt University School of Medicine, Nashville, TN, USA), and GST-p300 expression plasmids were obtained from $\mathrm{H} \mathrm{Lu}$ (Oregon Health and Science University, Portland, OR, USA) and D Livingston (Dana-Farber Cancer Institute, Boston, MA, USA).

\section{Protein interaction assays}

GST pull-down protein interaction assays were conducted as previously described (Stanojevic et al. 2004). Recombinant GST fusion protein input was determined and normalized by sodium dodecyl sulfate-polyacrylamide gel electrophoresis (SDS-PAGE) of fusion proteins that were synthesized in bacteria. Radiolabeled, in vitro translated proteins were synthesized from $p C M V \beta-p 300$ and pcDNA3-Bridge-1 plasmids with ${ }^{35}$ S-methionine in rabbit reticulocyte lysate kits purchased from Promega according to the manufacturer's protocol. GST protein interaction assays were conducted with glutathione Sepharose 4B beads (Amersham Biosciences, Piscataway, NJ, USA) as described (Stanojevic et al. 2004). Immunoprecipitation reactions were conducted with radiolabeled, in vitro translated ${ }^{35} \mathrm{~S}-$ Bridge- 1 and ${ }^{35} \mathrm{~S}-\mathrm{p} 300$ proteins and rabbit polyclonal anti-Bridge-1 or preimmune antiserum by previously reported methods (Thomas et al. 1999).

\section{Western blots}

Western blots were conducted by SDS-PAGE fractionation of whole-cell extracts prepared from transfected cells in 1 reporter lysis buffer (Promega) and electroblotting onto Immobilon-P membranes (Millipore, Bedford, MA, USA) (Thomas et al. 1999). Blots were incubated with primary rabbit polyclonal anti-Gal4 DBD antiserum (1:1000 dilution) (sc-577 from Santa Cruz Biotechnology, Santa Cruz, CA, USA) and secondary horseradish peroxidase-conjugated goat anti-rabbit antiserum (BioRad Laboratories, Richmond, CA, USA). Proteins were visualized by chemiluminescence with ECL Western blotting detection reagents (Amersham Life Sciences, Arlington Heights, IL, USA).

\section{Results}

\section{Histone deacetylase inhibition increases transcriptional} activation by Bridge-1

We previously identified an assay for studies of transcriptional activation by the coactivator Bridge- 1 in mammalian cells. In this transfection assay, a Gal4 DNAbinding domain-Bridge-1 fusion protein (Gal4-Bridge-1) activated a Gal4-reporter construct by as much as 30-fold as compared with the control Gal4 DNA-binding domain construct (Gal4) alone (Thomas et al. 1999). Using this transcriptional activation assay, we found that incubation with the histone deacetylase inhibitor trichostatin A increased the transcriptional activation by Bridge- 1 in a dose-dependent manner (Fig. 1A), suggesting that the regulation of transcriptional activation by Bridge- 1 is governed by the activity of histone-modifying enzymes within transcriptional regulatory complexes.

\section{Bridge-1 interacts with the histone acetyltransferase p300}

Because the histone acetyltransferase and nuclear receptor coactivator p300 is one of the principal coactivators implicated in the regulation of gene transcription in the endocrine pancreas, we conducted a series of studies to determine whether interactions between Bridge- 1 and p300 could contribute to the regulation of transcriptional activation by Bridge-1. First, we demonstrated that transcriptional activation of the Gal4-reporter construct by Gal4-Bridge-1 was augmented by the addition of exogenous p300 in vitro. In a dose-dependent manner, the addition of p300 to Gal4-Bridge-1 increased activation of the reporter by over 70-fold, an effect in marked excess of that observed for p300-mediated activation of the basal activity of the control Gal4 expression vector (Fig. 1B). These data suggest that p300 cooperates with Bridge- 1 to augment transcriptional activation.

To determine whether Bridge- 1 could directly interact with p300, we conducted a series of GST pull-down protein interaction assays. In these assays, we observed Bridge-1 interactions with p300 by combining GSTBridge-1 fusion proteins with ${ }^{35} \mathrm{~S}-l a b e l e d$, in vitro translated, full-length p300. Radiolabeled p300 strongly interacted with GST-Bridge-1, but not with the GST control protein (Fig. 1C). We also observed interactions between ${ }^{35}$ S-labeled, in vitro translated, full-length p300 and Bridge-1 proteins in immunoprecipitation assays (Fig. 1D). In these studies, anti-Bridge-1 antiserum directed against a midmolecule epitope within Bridge-1 (Thomas et al. 1999), but not preimmune antiserum, immunoprecipitated p300 in combination with Bridge-1.

The coactivator p300 is a large modular protein with multiple interaction domains, including cysteine-histidinerich regions known as $\mathrm{C} / \mathrm{H}$ domains (Chakravarti et al. 1996) (Fig. 1E, upper panel). To identify the regions within p300 that interacted with Bridge-1, we combined ${ }^{35} \mathrm{~S}-\mathrm{lab}$ eled, in vitro translated Bridge-1 with GST-p300 fusion proteins that spanned the three $\mathrm{C} / \mathrm{H}$ protein interaction domains within $\mathrm{p} 300$. We identified strong interactions between Bridge- 1 and GST-p300 fusion protein segments encompassing p300 amino acids 1-595 and 744-1571, but not with the GST-p300 fusion protein 
A.

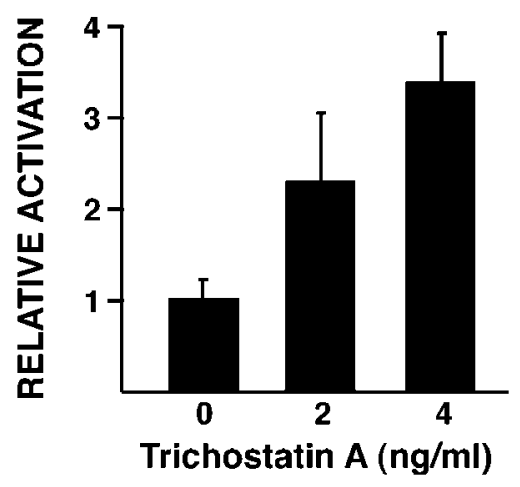

C.

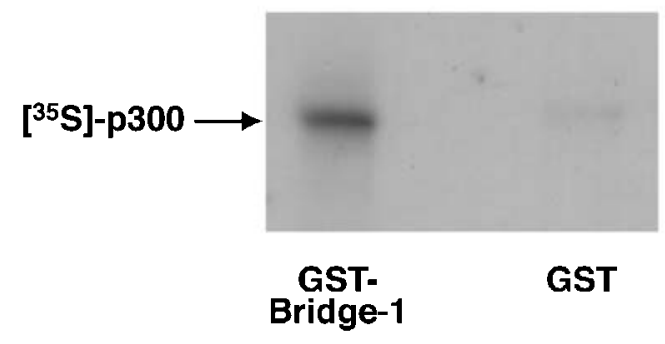

B.

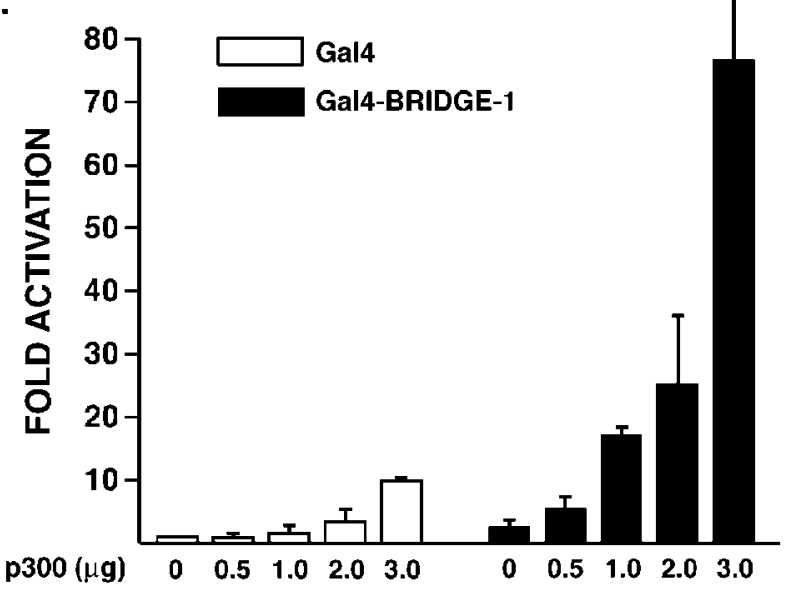

D.

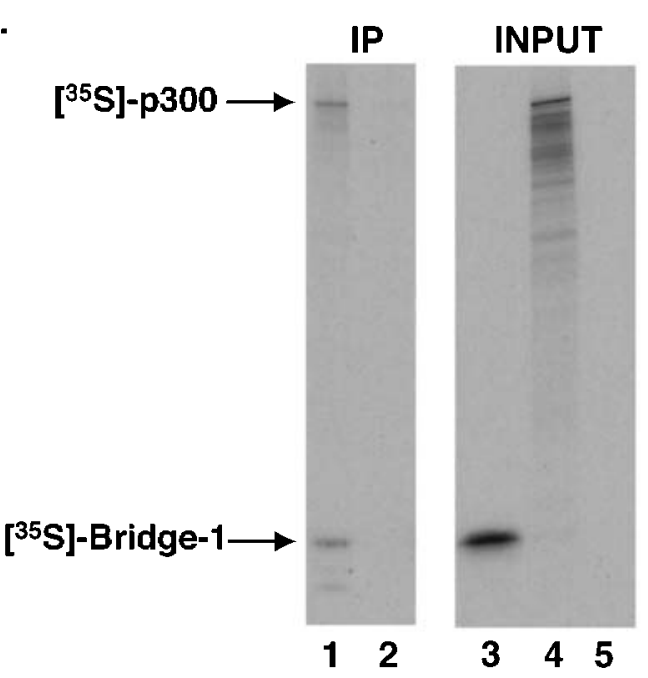

E.

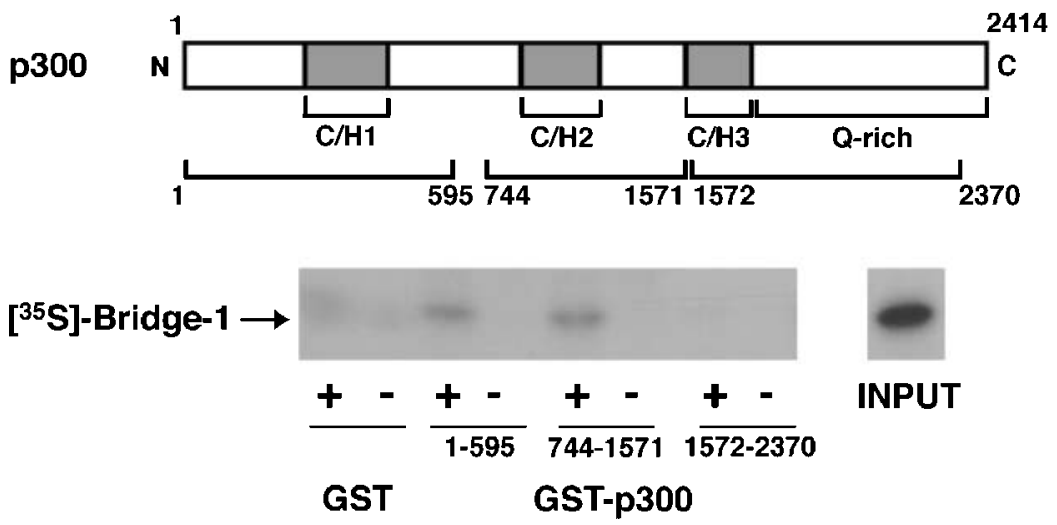


encompassing amino acids 1572-2370 (Fig. 1E, lower panel). These results indicate that Bridge- 1 can interact with multiple regions within $\mathrm{p} 300$, including those encompassing the $\mathrm{C} / \mathrm{H} 1$ and $\mathrm{C} / \mathrm{H} 2$ protein interaction domains. The histone acetyltransferase domain of p300 is included within amino acids 744-1571 (Chan \& La Thangue 2001). We noted that Bridge-1 did not directly interact with the carboxy-terminal segment of p300 that is implicated in interactions with the insulin gene regulators PDX-1 and NeuroD1/Beta-2 (Qiu et al. 1998, 2002, Stanojevic et al. 2004).

\section{Sequestration of $p 300$ by E1A impairs transcriptional activation by Bridge-1}

To determine whether endogenous p300 regulates the extent of transcriptional activation by Bridge-1, we overexpressed the exogenous adenoviral protein E1A to sequester p300 (Stein et al. 1990, Qiu et al. 1998). The expression of increasing amounts of E1A decreased the transcriptional activation of a Gal4-CAT reporter construct by Gal4-Bridge-1 (Fig. 2A) while preserving the expression of the transfected Gal4-Bridge-1 fusion proteins on Western blots (data not shown). Although exogenous E1A reduced transcriptional activation by Bridge-1, a mutant E1A protein (E1A $\Delta 2-36)$ that is unable to bind and sequester p300 (Stein et al. 1990) did not interfere with transcriptional activation (Fig. 2B). Collectively, these data suggest that transcriptional activation by Bridge- 1 depends, in part, on the availability of p300.

\section{$P D Z$ and carboxy-terminal domains within Bridge-1 are required for transcriptional activation}

To identify domains within Bridge- 1 that are required for transcriptional activation, we generated Bridge-1 deletion mutants and assessed their function in both yeast and mammalian cells. Bridge-1 segments containing amino acids $1-72$ or $1-132$, which lack carboxy-terminal
A.

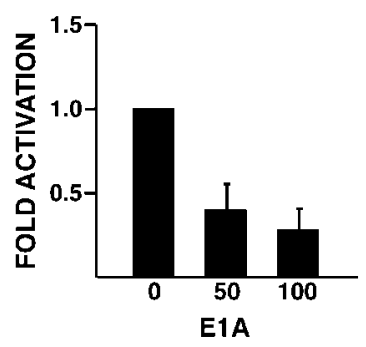

B.

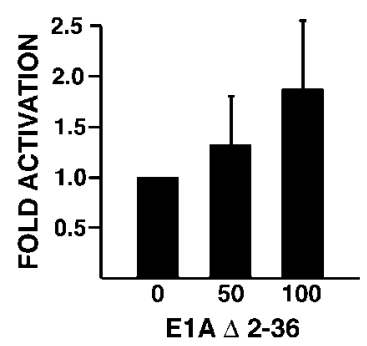

Figure 2 Sequestration of $\mathrm{p} 300$ by the adenoviral protein E1A interferes with transcriptional activation by Bridge-1. (A)

Transcriptional activation by Bridge- 1 is suppressed by E1A. BHK cells were transfected in duplicate with $1 \mu \mathrm{g}$ Gal4-Bridge- 1 and 0 , 50, or $100 \mathrm{ng} \mathrm{E} 1 \mathrm{~A}$ and $1 \mu \mathrm{g}$ Gal4-CAT reporter. pBluescript was added to normalize the total amount of DNA for all conditions. Results shown are the mean relative activation levels derived from two independent transfections, normalized to the activity of Gal-Bridge- 1 set at $1 \cdot 0$. (B) Mutant E1A does not interfere with transcriptional activation by Bridge- 1 . BHK cells were transfected in duplicate with $1 \mu \mathrm{g}$ Gal4-Bridge- 1 and 0, 50, or 100 ng mutant $\mathrm{E} 1 \mathrm{~A}(\Delta 2-36)$ and $1 \mu \mathrm{g}$ Gal4-CAT reporter. Results shown are the mean relative activation levels derived from two independent transfections, normalized to the activity of Gal-Bridge- 1 set at $1 \cdot 0$.

portions of the protein that encompass the PDZ domain, had markedly impaired transcriptional activation in yeast cells of $4-11 \%$ of the wild-type Bridge- 1 activity (Fig. 3A). Similarly, in mammalian cells, the levels of activation of the Gal4-reporter construct by Gal4Bridge- 1 mutant proteins encoding amino acids $1-72$ or 1-133 were nearly undetectable (Fig. 3B), despite comparable protein expression of transfected wild-type and mutant Bridge-1 fusion proteins, as determined by Western blots (Fig. 3C). Therefore, the carboxy-terminal segment of Bridge-1 that includes the PDZ domain is required for transcriptional activation.

To determine the importance of the PDZ domain within Bridge-1 for transcriptional activation, we introduced proline substitution point mutations within several

\footnotetext{
Figure 1 The coactivator Bridge-1 interacts with multiple domains of p300. (A) Trichostatin A increases transcriptional activation by Bridge-1. BHK cells were transfected in duplicate with $1 \cdot 5 \mu \mathrm{g}$ Gal4-Bridge- 1 or empty Gal4 expression vector, $3 \cdot 25 \mu \mathrm{g}$ pBluescript and $250 \mathrm{ng}$ of Gal4-luciferase reporter and treated with vehicle (0), 2, or $4 \mathrm{ng} / \mathrm{ml}$ trichostatin A for 24 h. Results shown are the mean \pm S.E.M. relative luciferase activity of Gal4-Bridge- 1 normalized to the empty Gal4 expression vector ( $n=3-5$ transfections). (B) p300 increases the transcriptional activation by Bridge- 1 . BHK cells were transfected in duplicate with $0-3 \mu \mathrm{g}$ of the p300 expression vector pCMV-p300 and $0.5 \mu \mathrm{g}$ Gal4-Bridge- 1 or empty Gal4 expression vector and $1 \mu \mathrm{g}$ of Gal4-CAT reporter. pBluescript was used to normalize the total DNA content for all conditions. Data shown are the mean of two transfections. (C) Bridge-1 directly interacts with p300. GST-Bridge- 1 or GST control proteins were incubated with in vitro translated, ${ }^{35} \mathrm{~S}$-p 300 in GST protein interaction assays. A representative autoradiogram is shown of an SDS-polyacrylamide gel from a GST pull-down protein interaction assay with the migration position of ${ }^{35}$ S-p300 designated (arrow). (D) Coimmunoprecipitation of ${ }^{35}$ S-Bridge- 1 and ${ }^{35} \mathrm{~S}-\mathrm{p} 300$. Rabbit polyclonal anti-Bridge-1 antiserum (lane 1), but not preimmune antiserum (lane 2) immunoprecipitated ${ }^{35} \mathrm{~S}$-p 300 with ${ }^{35} \mathrm{~S}$-Bridge-1, as shown by autoradiography (left panel, IP, immunoprecipitation). $10 \%$ input of in vitro translated ${ }^{35} \mathrm{~S}$-Bridge-1 (lane 3) and ${ }^{35} \mathrm{~S}-\mathrm{p} 300$ (lane 4 ) proteins used in the immunoprecipitation reactions and $10 \%$ of the empty vector pcDNA3 lysate control reaction (lane 5) are shown by autoradiography (right panel, INPUT). (E) Bridge-1 interacts with multiple domains within p300. A schematic diagram depicts protein interaction domains within p300 (adapted from Chakravarti et al. 1996) (upper panel). In vitro translated, ${ }^{35}$ S-Bridge- 1 (+) or empty vector control in vitro transcription and translation reactions (-) were incubated with GST-p300 (1-595), GST-p300 (744-1571), GST-p300 (1572-2370) or GST control (GST) proteins in GST protein interaction assays. Representative autoradiograms of SDS-polyacrylamide gels from a GST pull-down protein interaction assay and of $10 \%$ input of the ${ }^{35}$ S-Bridge- 1 are shown (lower panel).
} 
A.

\section{YEAST CELLS}
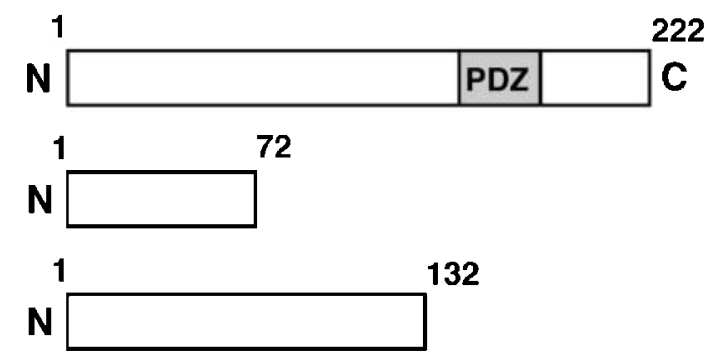
132

\section{MAMMALIAN CELLS}
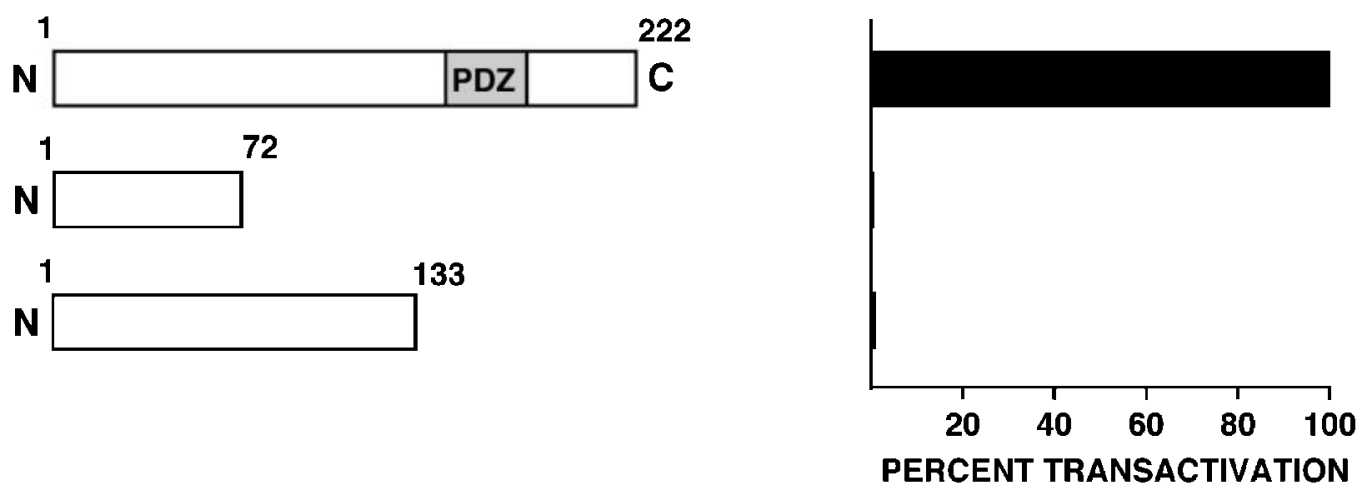

c.

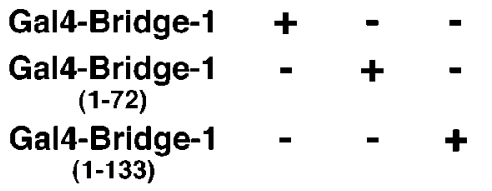


conserved amino acids of the PDZ domain of Bridge-1. As compared with the wild-type Gal4-Bridge-1 fusion protein, the mutant Gal4-Bridge-1 fusion proteins V159P, V164P, V175P, D156P and G151P all demonstrated severe impairment in the activation of the Gal4-reporter construct (Fig. 4A), despite evidence of sufficient protein expression levels (Fig. 4B). We noted the most severe impairment in transcriptional activation in the Gal4-Bridge-1 fusion protein D156P. The level of transcriptional activation of the Gal4-reporter construct by Gal4-Bridge-1 (D156P) was approximately 10\% of that observed with expression of the wild-type Gal4-Bridge-1 fusion protein (Fig. 4C).

Deletion or mutagenesis of the Bridge-1 PDZ domain disrupts interactions with $\mathrm{p} 300$

Because our transfection data suggested that transcriptional activation by Bridge- 1 partially depends on the availability of p300, we wondered whether mutations that reduce transcriptional activation by Bridge- 1 might also disrupt the interactions of Bridge- 1 with p300. The deletion of carboxy-terminal segments of Bridge-1 that include the PDZ domain to generate GST-Bridge-1 fusion proteins encoding Bridge-1 amino acids 1-72 and 1-133 interfered with interactions with ${ }^{35}$ S-labeled, in vitro translated p300 in GST pull-down protein interaction assays (Fig. 4D). Similarly, the mutant GST-Bridge-1 fusion protein D156P did not interact with radiolabeled wild-type p300, in contrast to the wild-type GST-Bridge-1 fusion protein (Fig. 4E). Thus, mutations of Bridge-1 that disrupted transcriptional activation also interfered with interactions with p300.

\section{Discussion}

In this report we identify the PDZ-domain coactivator of insulin gene expression Bridge- 1 as an interaction partner for the nuclear receptor coactivator p300. The coactivator Bridge-1 is likely to regulate target gene transcription by participating in multiple protein interactions to enhance transcriptional activation. Although our previous studies of Bridge-1-regulated transcriptional activation focused on the insulin promoter (Thomas et al. 1999), the broad distribution of the expression patterns for both p300 and
Bridge- 1 suggests that these two coregulators may regulate a variety of target genes in cellular contexts beyond the endocrine pancreas. The appropriate assembly of multiprotein complexes is essential for the precise regulation of transcriptional activation of complex promoters. In these studies, we demonstrate that transcriptional activation by Bridge- 1 depends not only on the availability of endogenous p300 but also on structural features of the Bridge-1 PDZ domain that are required for interactions with p300.

We propose that Bridge-1 may participate in target gene regulation by contributing to multiprotein complexes to couple interactions with p300 and interactions with other transcriptional regulatory proteins (Fig. 5). The observed capacity of Bridge-1 to interact with multiple domains of p300 could provide opportunities for multifocal and combinatorial protein interactions with increased complexity and flexibility for the regulation of target genes by Bridge-1. For example, because Bridge- 1 interacts with regions of p300 distinct from those required for p300 interactions with PDX-1 and NeuroD1/Beta-2, a single p300 molecule could serve as a scaffold to promote and stabilize the simultaneous assembly of multiple insulin gene regulators with Bridge- 1 to increase insulin production. Furthermore, p300 has tumor-suppressor properties, and we recently identified Bridge- 1 as a potential regulator of pancreatic beta-cell mass in a transgenic mouse model of Bridge-1 overexpression (Volinic et al. 2006). Additional studies will be needed to determine whether Bridge- 1 and p300 interactions influence the regulation of cellular replication and/or apoptosis in certain physiologic settings.

Rat Bridge-1 is homologous to the human protein PSMD9 that was isolated in a complex with a proteasomal subunit called TBP-1/Rpt5 that is known to have transcriptional regulatory properties (Ohana et al. 1993, DeMartino et al. 1996, Watanabe et al. 1998, Thomas et al. 1999, Gonzales et al. 2002). It is interesting to note that p300 can participate in similar protein regulatory complexes, as p300 protein expression levels are known to be regulated by the $26 \mathrm{~S}$ proteasome under certain experimental conditions, including treatment with doxorubicin, sodium butyrate or inhibitors of PI3 kinase (Poizat et al. 2000, Li et al. 2002, Chen et al. 2004). As a potential modulator of the function of the $26 \mathrm{~S}$ proteasome, Bridge- 1 interactions with p300 could conceivably facilitate the coupling of the intrinsic ubiquitin ligase activity of $\mathrm{p} 300$ (Grossman et al. 2003) with proteasomal degradation

\footnotetext{
Figure 3 The carboxy-terminal domains of Bridge- 1 are required for transcriptional activation. (A) Deletion of carboxy-terminal domains of Bridge-1 reduces transcriptional activation in yeast. Quantitative beta-galactosidase assays were conducted on yeast transformed with LexA-Bridge-1, LexA-Bridge-1(1-72), or LexA-Bridge-1(1-132) expression vectors in conjunction with a LexA-operator-beta-galactosidase reporter. Results shown are the mean of two independent transformations, normalized to the activity of LexA-Bridge- 1 set at $100 \%$. (B) Deletion of carboxy-terminal domains of Bridge-1 reduces transcriptional activation in mammalian cells. BHK cells were transfected in duplicate with 1.5 $\mu \mathrm{g}$ Gal4-Bridge-1, Gal4-Bridge-1 (1-72), or Gal4-Bridge-1 (1-133), 3.25 $\mu \mathrm{g}$ pBluescript, and 250 ng Gal4-luciferase reporter. Results shown are the mean of five transfections, normalized to the activity of Gal4-Bridge-1 set at $100 \%$. (C) Relative protein expression levels of Gal4-Bridge-1 deletion mutants on a Western blot of extracts from BHK cells transfected with Gal4-Bridge-1, Gal4-Bridge-1 (1-72) or Gal4-Bridge-1 (1-133), as indicated (+), conducted with anti-Gal4 antiserum.
} 
A.

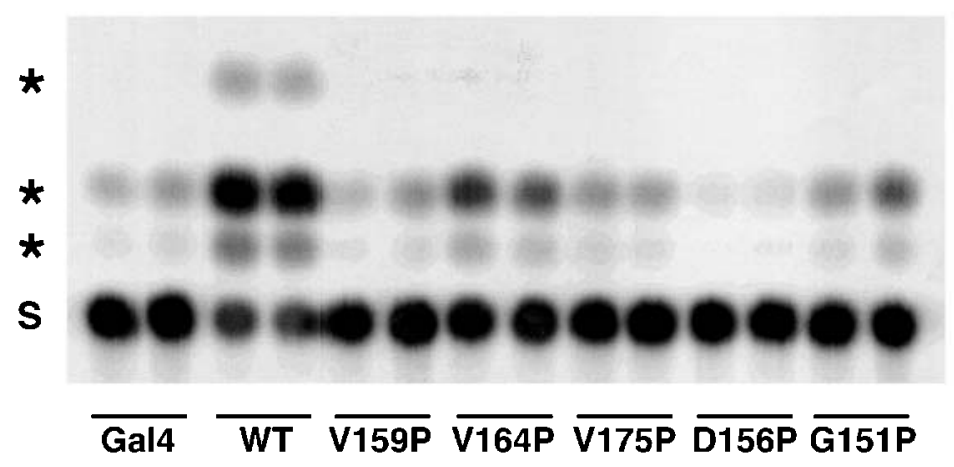

B.
1) $W T$
2) G151P
3) D156P
4) V159P
5) V164P
6) V175P

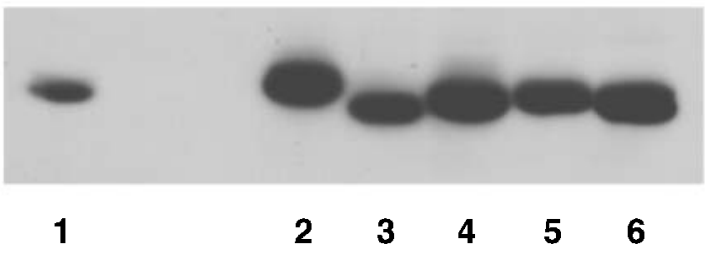

C.

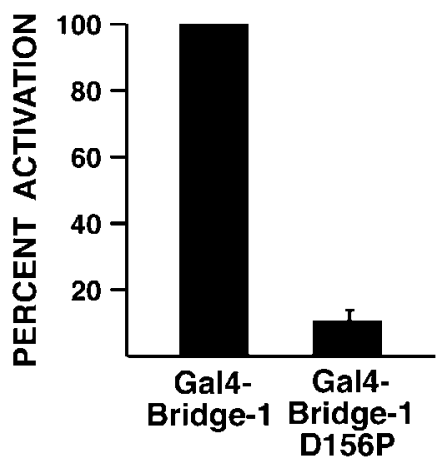

D.
1) GST-Bridge 1
2) GST
3) GST-Bridge-1
4) GST-Bridge-1

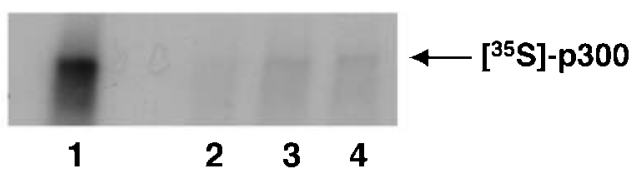
(1-133)

E.

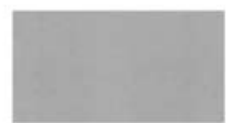

GST

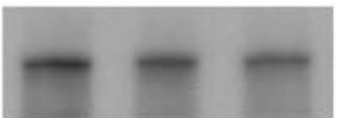

GST-Bridge-1

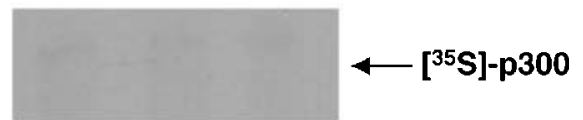

GST-Bridge-1
(D156P) 


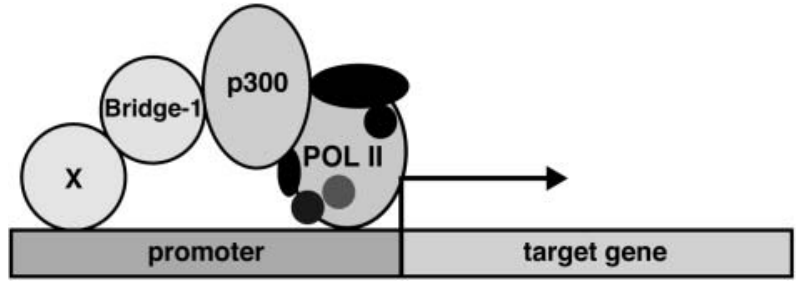

Figure 5 Proposed model of Bridge- 1 interactions with p300 in the activation of target gene transcription. In this schematic diagram, p300 interacts with Bridge- 1 in multiprotein complexes coupled to the basal transcription machinery (POL II). We propose that Bridge- 1 recruits other transcriptional regulators (designated by $\mathrm{X}$ ) to $\mathrm{p} 300$-containing protein complexes to activate target gene transcription.

machinery in the regulation of the expression levels of p300-interacting proteins (Grossman et al. 1998).

Although multiple interacting proteins for p300 have been identified, we note increasing evidence that the specific composition of coregulator transcriptional complexes is of physiologic and therapeutic relevance. For example, differing biologic actions of ligands for nuclear hormone receptors may be mediated by their alteration of the composition of associated coregulatory proteins on target gene promoters (Savkur et al. 2004). In particular, the therapeutic utility of selective estrogen receptor modulators in directing biologic effects to distinct cell types may be directly regulated to differences in coregulator recruitment (Shang \& Brown 2002). Future studies will be needed to identify the physiologic and pathophysiologic importance of the participation of Bridge- 1 in regulatory complexes with p300 in different experimental contexts, including the regulation of insulin production in diabetes and the modulation of rates of cellular replication and/or apoptosis. Although we identified the insulin gene as the first proposed target of Bridge-1 regulation, it is likely that this coactivator participates in modulating the expression levels of a broad repertoire of additional genes. These studies indicate that the regulation of the transcriptional activation of target gene expression by the coactivator Bridge-1 may rely substantially on the availability of p300.

\section{Acknowledgements}

We thank Joel Habener and our colleagues in the Molecular Endocrinology Unit for their support and interest in these studies. We acknowledge expert technical assistance from Wai-Ying Leung, Josephine Ngai and Matthew Tenser, and we thank D Livingston, $\mathrm{H} \mathrm{Lu}$ and $\mathrm{R}$ Stein for their generous gifts of reagents. We appreciate the expert assistance of Kimberly MacDonald in the preparation of the manuscript.

\section{Funding}

These studies were supported by grants from the National Institutes of Health (DK02476 and DK59419) to M K T. The authors declare that there is no conflict of interest that would prejudice this paper.

\section{References}

Andreassen OA, Dedeoglu A, Stanojevic V, Hughes DB, Browne SE, Leech CA, Ferrante RJ, Habener JF, Beal MF \& Thomas MK 2002 Huntington's disease of the endocrine pancreas: insulin deficiency and diabetes mellitus due to impaired insulin gene expression. Neurobiology of Disease 11 410-424.

Chakrabarti SK, Francis J, Ziesmann SM, Garmey JC \& Mirmira RG 2003 Covalent histone modifications underlie the developmental regulation of insulin gene transcription in pancreatic beta cells. Journal of Biological Chemistry 278 23617-23623.

Chakravarti D, LaMorte VJ, Nelson MC, Nakajima T, Schulman IG, Juguilon H, Montminy M \& Evans RM 1996 Role of CBP/P300 in nuclear receptor signalling. Nature 383 99-103.

Chan HM \& La Thangue NB 2001 p300/CBP proteins: HATs for transcriptional bridges and scaffolds. Journal of Cell Science $\mathbf{1 1 4}$ 2363-2373.

Chen J, Halappanavar SS, St-Germain JR, Tsang BK \& Li Q 2004 Role of Akt/protein kinase B in the activity of transcriptional coactivator p300. Cellular and Molecular Life Sciences 61 1675-1683.

Figure 4 Mutagenesis of the PDZ domain of Bridge- 1 disrupts transcriptional activation. (A) Point mutations within conserved amino acids of the Bridge-1 PDZ domain disrupt transcriptional activation. BHK cells were transfected in duplicate with $4 \mu \mathrm{g}$ Gal4-Bridge-1 (WT), Gal4-Bridge-1 (V159P), Gal4-Bridge-1 (V164P), Gal4-Bridge-1 (V175P), Gal4-Bridge-1 (D156P), Gal4-Bridge-1 (G151P) or empty Gal4 expression vector $(\mathrm{Gal} 4)$, as indicated, with $1 \mu \mathrm{g}$ Gal4-CAT reporter. A fluorescence image of a thin-layer chromatogram from a representative CAT assay is shown with the fluorescent acetylated products $\left(^{*}\right)$ and substrate (S) indicated. (B) Relative Gal4-Bridge-1 mutant protein expression levels are shown on a Western blot of extracts from BHK cells transfected with Gal4-Bridge-1 (WT), Gal4-Bridge-1 (V159P), Gal4-Bridge-1 (V164P), Gal4-Bridge-1 (V175P), Gal4-Bridge-1 (D156P) or Gal4-Bridge-1 (G151P), as indicated, conducted with anti-Gal4 antiserum. (C) The Bridge-1 (D156P) mutant has diminished transcriptional activation. BHK cells were transfected in duplicate with $4 \mu \mathrm{g}$ Gal4-Bridge-1 or Gal4-Bridge-1 (D156P), as indicated, with $1 \mu \mathrm{g}$ Gal4-CAT reporter. Results shown are the mean \pm S.E.M. of three transfections, normalized to the activity of Gal4-Bridge- 1 set at $100 \%$. (D) Deletion of the PDZ domain decreases Bridge-1 interactions with p300. GST-Bridge-1, GST-Bridge-1 (1-72), GST-Bridge-1 (1-133) or GST control (GST) proteins were incubated with in vitro translated ${ }^{35} \mathrm{~S}-\mathrm{p} 300$ in GST pull-down protein interaction assays. A representative autoradiogram is shown of an SDS-polyacrylamide gel from a GST pull-down protein interaction assay with the migration position of ${ }^{35} \mathrm{~S}$-p 300 (arrow) indicated. (E) Mutagenesis of the PDZ domain decreases Bridge-1 interactions with p300. GST-Bridge-1, GST-Bridge-1 (D156P) or GST control (GST) proteins were incubated with in vitro translated ${ }^{35} \mathrm{~S}-\mathrm{p} 300$ in GST protein interaction assays in duplicate or triplicate samples. A representative autoradiogram is shown of an SDS-polyacrylamide gel from a GST pull-down protein interaction assay with the migration position of ${ }^{35} \mathrm{~S}-\mathrm{p} 300$ (arrow) indicated. 
DeMartino GN, Proske RJ, Moomaw CR, Strong AA, Song X, Hisamatsu H, Tanaka K \& Slaughter CA 1996 Identification, purification, and characterization of a PA700-dependent activator of the proteasome. Journal of Biological Chemistry 271 3112-3118.

Eeckhoute J, Formstecher P \& Laine B 2001 Maturity-onset diabetes of the young type 1 (MODY1)-associated mutations R154X and E2767Q in hepatocyte nuclear factor 4 alpha (HNF4 alpha) gene impair recruitment of $\mathrm{p} 300$, a key transcriptional coactivator. Molecular Endocrinology 15 1200-1210.

Fajans SS, Bell GI \& Polonsky KS 2001 Molecular mechanisms and clinical pathophysiology of maturity-onset diabetes of the young. New England Journal of Medicine 345 971-980.

Giles RH, Peters DJ \& Breuning MH 1998 Conjunction dysfunction: $\mathrm{CBP} / \mathrm{p} 300$ in human disease. Trends in Genetics 14 178-183.

Golemis EA, Gyuris J \& Brent R 1994 Interaction trap/two-hybrid system to identify interacting proteins, unit $13 \cdot 14$. In Current Protocols in Molecular Biology, pp 13·14·11-13·14·17, Eds FM Ausubel, R Brent, RE Kingston, DD Moore, JG Seidman, JA Smith \& K Struhl. New York: Wiley.

Gonzales F, Delahodde A, Kodadek T \& Johnston SA 2002 Recruitment of a $19 \mathrm{~S}$ proteasome subcomplex to an activated promoter. Science 296 548-550.

Grossman SR, Deato ME, Brignone C, Chan HM, King AL, Tagami H, Nakatani Y \& Livingston DM 2003 Polyubiquitination of p53 by a ubiquitin ligase activity of p300. Science 300 342-344.

Grossman SR, Perez M, Kung AL, Joseph M, Mansur C, Xiao ZX, Kumar S, Howley PM \& Livingston DM 1998 p300/MDM2 complexes participate in MDM2-mediated p53 degradation. Molecular Cell 2 405-415.

Iyer NG, Ozdag H \& Caldas C 2004 p300/CBP and cancer. Oncogene $234225-4231$.

Kurihara N, Ishizuka S, Demulder A, Menaa C \& Roodman GD 2004 Paget's disease - a VDR coactivator disease? Journal of Steroid Biochemistry and Molecular Biology 89-90 321-325.

Li Q, Su A, Chen J, Lefebvre YA \& Hache RJ 2002 Attenuation of glucocorticoid signaling through targeted degradation of p300 via the 26S proteasome pathway. Molecular Endocrinology 16 2819-2827.

Malecki MT, Jhala US, Antonellis A, Fields L, Doria A, Orban T, Saad M, Warram JH, Montminy M \& Krolewski AS 1999 Mutations in NEUROD1 are associated with the development of type 2 diabetes mellitus. Nature Genetics 23 323-328.

Mosley AL, Corbett JA \& Ozcan S 2004 Glucose regulation of insulin gene expression requires the recruitment of $\mathrm{p} 300$ by the beta-cell-specific transcription factor Pdx-1. Molecular Endocrinology 18 2279-2290.

Ohana B, Moore PA, Ruben SM, Southgate CD, Green MR \& Rosen CA 1993 The type 1 human immunodeficiency virus Tat binding protein is a transcriptional activator belonging to an additional family of evolutionarily conserved genes. PNAS $\mathbf{9 0}$ $138-142$.

Poizat C, Sartorelli V, Chung G, Kloner RA \& Kedes L 2000 Proteasome-mediated degradation of the coactivator p300 impairs cardiac transcription. Molecular and Cellular Biology 20 8643-8654.

Qiu Y, Sharma A \& Stein R 1998 p300 mediates transcriptional stimulation by the basic helix-loop-helix activators of the insulin gene. Molecular and Cellular Biology 18 2957-2964.
Qiu Y, Guo M, Huang S \& Stein R 2002 Insulin gene transcription is mediated by interactions between the p300 coactivator and PDX-1, BETA2, and E47. Molecular and Cellular Biology 22 $412-420$.

Roelfsema JH, White SJ, Ariyurek Y, Bartholdi D, Niedrist D, Papadia F, Bacino CA, den Dunnen JT, van Ommen GJ, Breuning $\mathrm{MH}$ et al. 2005 Genetic heterogeneity in Rubinstein-Taybi syndrome: mutations in both the CBP and EP300 genes cause disease. American Journal of Human Genetics 76 572-580.

Savkur RS, Bramlett KS, Clawson D \& Burris TP 2004 Pharmacology of nuclear receptor-coregulator recognition. Vitamins and Hormones 68 145-183.

Shang Y \& Brown M 2002 Molecular determinants for the tissue specificity of SERMs. Science 295 2465-2468.

Sharma A, Moore M, Marcora E, Lee JE, Qiu Y, Samaras S \& Stein R 1999 The NeuroD1/BETA2 sequences essential for insulin gene transcription colocalize with those necessary for neurogenesis and p300/CREB binding protein binding. Molecular and Cellular Biology $19704-713$.

Stanojevic V, Habener JF \& Thomas MK 2004 Pancreas duodenum homeobox-1 transcriptional activation requires interactions with p300. Endocrinology 145 2918-2928.

Stanojevic V, Yao KM \& Thomas MK 2005 The coactivator Bridge-1 increases transcriptional activation by pancreas duodenum homeobox-1 (PDX-1). Molecular Cellular Endocrinology 237 67-74.

Stein RW, Corrigan M, Yaciuk P, Whelan J \& Moran E 1990 Analysis of E1A-mediated growth regulation functions: binding of the 300-kilodalton cellular product correlates with E1A enhancer repression function and DNA synthesis-inducing activity. Journal of Virology 64 4421-4427.

Thomas MK, Yao KM, Tenser MS, Wong GG \& Habener JF 1999 Bridge-1, a novel PDZ-domain coactivator of E2A-mediated regulation of insulin gene transcription. Molecular and Cellular Biology 19 8492-8504.

Vo N \& Goodman RH 2001 CREB-binding protein and p300 in transcriptional regulation. Journal of Biological Chemistry 276 13505-13508.

Volinic J, Lee JH, Eto K, Kaur V \& Thomas MK 2006 Overexpression of the coactivator Bridge-1 results in insulin deficiency and diabetes. Molecular Endocrinology (2005 Aug 11 [Epub ahead of print]).

Watanabe TK, Saito A, Suzuki M, Fujiwara T, Takahashi E, Slaughter CA, DeMartino GN, Hendil KB, Chung CH, Tanahashi $\mathrm{N}$ et al. 1998 cDNA cloning and characterization of a human proteasomal modulator subunit, p27. Genomics 50 241-250.

Yao TP, Oh SP, Fuchs M, Zhou ND, Ch'ng LE, Newsome D, Bronson RT, Li E, Livingston DM \& Eckner R 1998 Gene dosage-dependent embryonic development and proliferation defects in mice lacking the transcriptional integrator p300. Cell 93 361-372.

Received 20 April 2005

Accepted 16 August 2005

Made available online as an Accepted Preprint

8 September 2005 